\title{
Optimal Filtering Algorithm implementation in FPGAs for the ATLAS TileCal Read-Out Drivers
}

\section{Pablo Moreno}

IFIC (CSIC \& Universitat de Valencia)

On behalf of the ATLAS Tile Calorimeter System

\section{The ATLAS Tile Calorimeter}

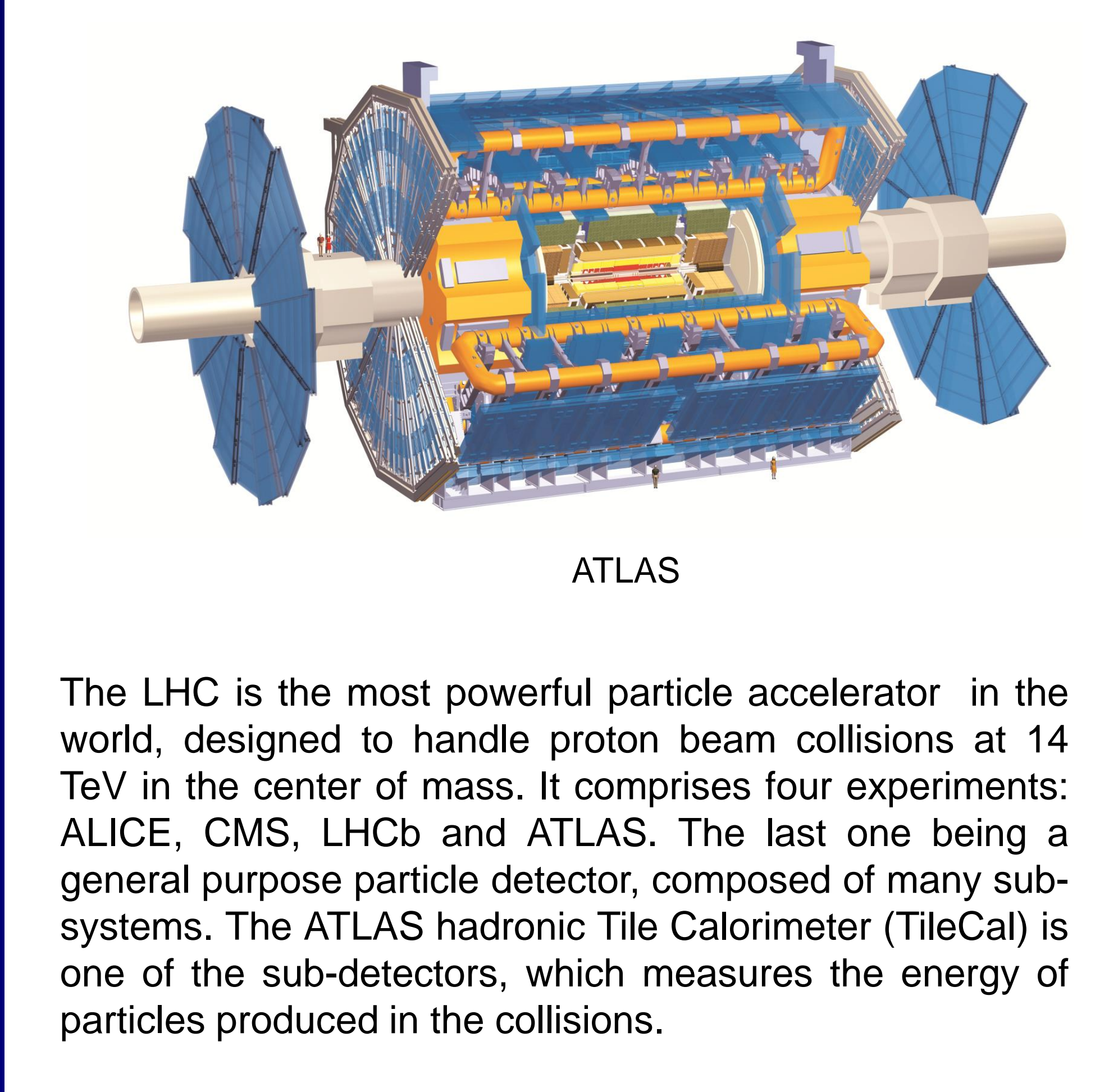

ATLAS TDAQ

The Trigger and Data Acquisition System (TDAQ) is the software that manages the hardware resources for the read-out and the trigger of the detector. It operates on an domains in the dataflow, called levels of trigger.

Front-End Electronics

TileCal is based on scintillating plastic tiles arranged in cells that produce light in the interaction with the particles. The light produced in a cell is converted to an electrical pulse in a photomultiplier tube (PMT), which is

and read out, forming a TileCal read-out channel. barrel partitions (EBA, LBA, LBC and EBC), each containing 64 modules that hold up to 48 PMTs. The readout of the whole calorimeter consists of 9856 channels
that are serialized and transmitted at $680 \mathrm{Mbps}$, using radiation-tolerant and is placed in a separated cavern.
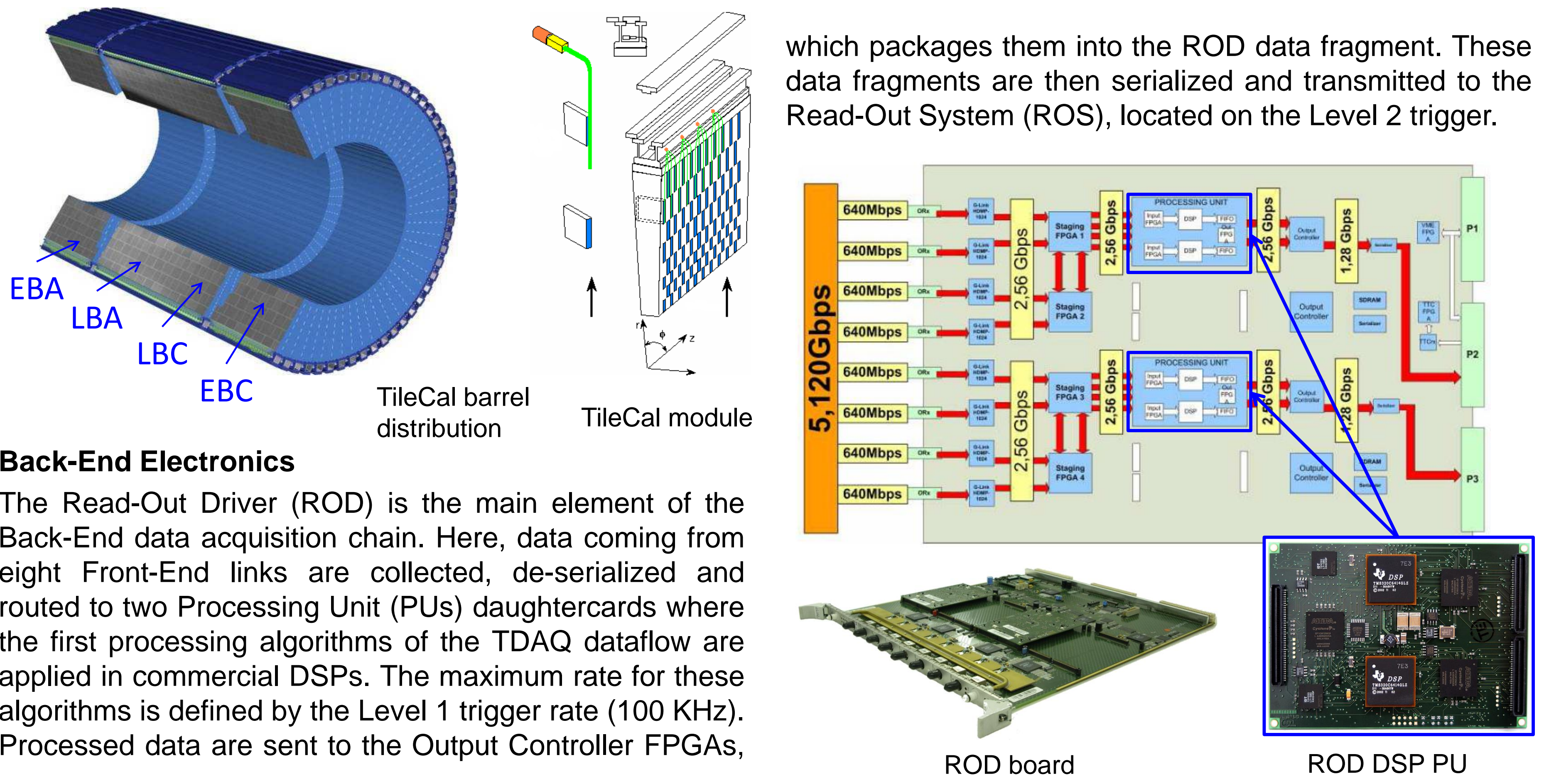

\section{Motivation}

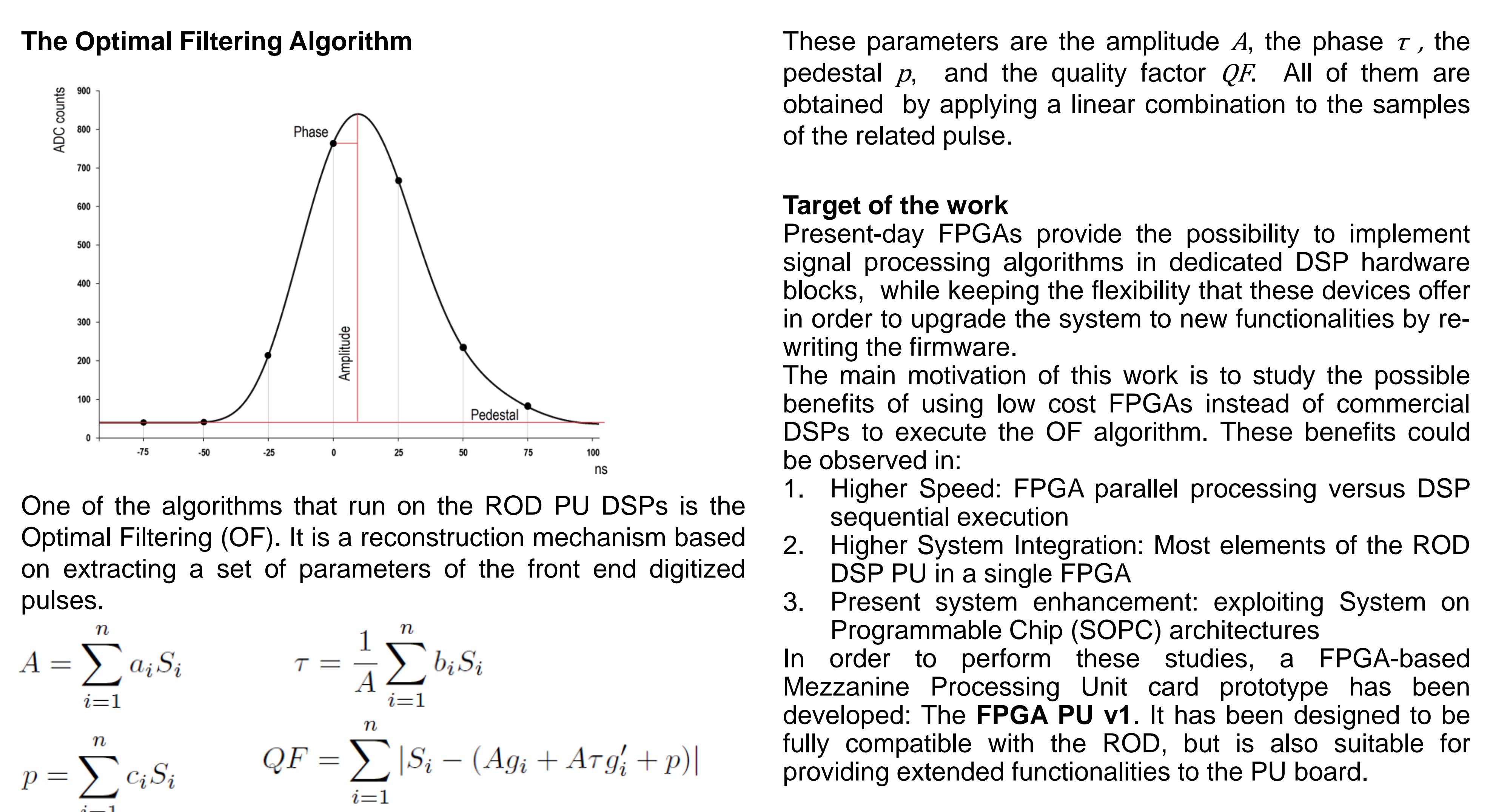

\section{Hardware Development}

Functional Description

Data for the OF algorithm enter through the Mezzanine connectors $A$ and $B$. The algorithm
processing takes place in the FPGA, a 484 -in processing takes place
ALTERA Cyclone III. Finally, the output of this processing task is sent through the Mezzanine

ALTERA Cyclone III EP3C40

- 484 pin BGA (331 user IOs)

39600 Logic Elements: - -input IUT + FF Register

$126 \times$ medded malipliers: $18 \times 18$ bit operation

$-126 \times 9 \times 10$

- sOPC support (NIOS, ARM, Frescale)

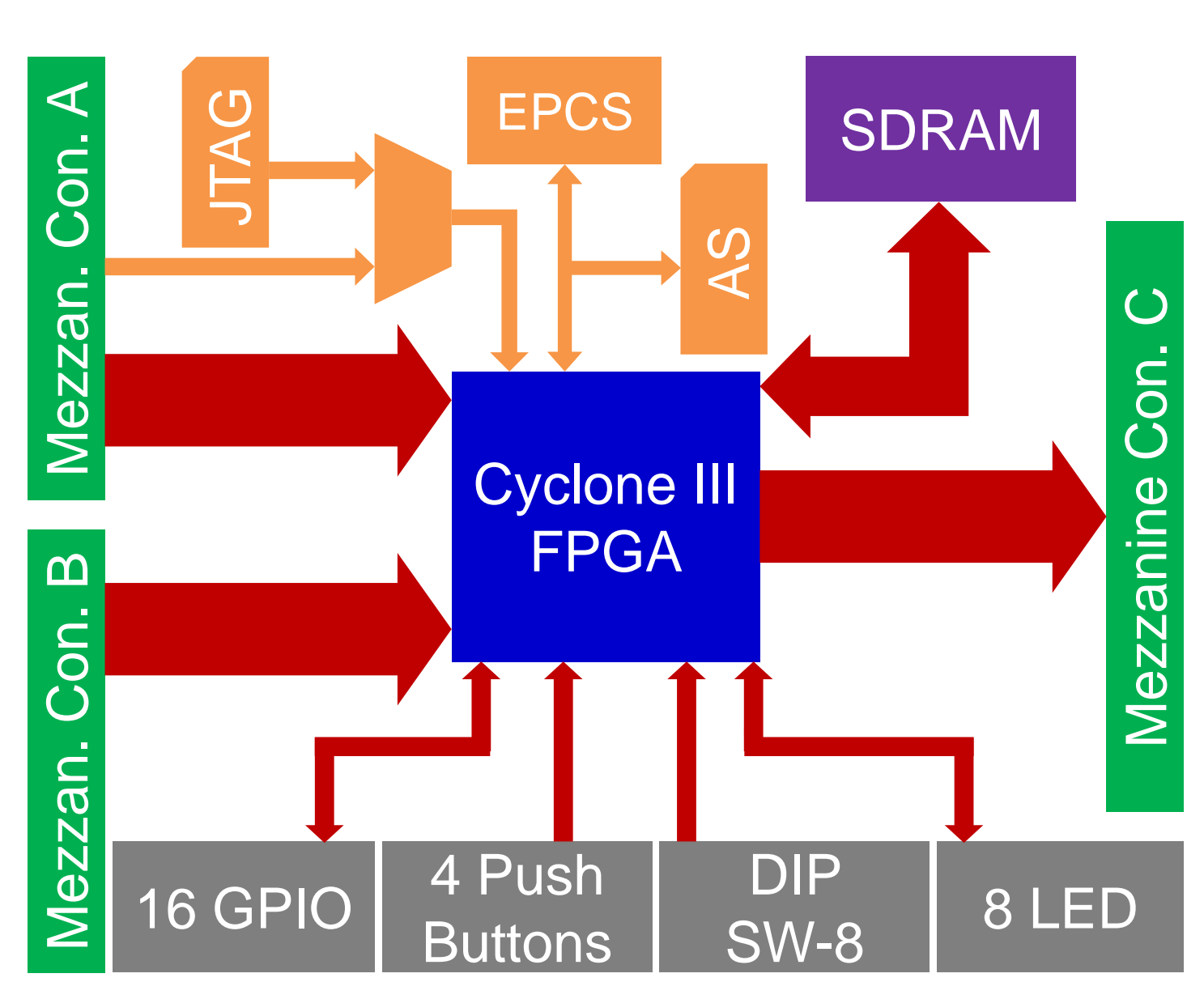

The FPGA implements different contiguration schemes: JTAG configures the FPGA directly, while Active Serial (AS) uses the data stored on a Flash memory (EPCS) to configure the device on every system power-up. The protolype hosts a set of user interfaces (SWithes, pushibutions, LEDs, etc.) for debugging purposes. A 128 Mbit SDRAM has been also placed on the PCB. Combined with an embedded sof

Power Distribution

Four different supplies are needed for powering the FPGA. Therefore the design requires a $2.5 \mathrm{~V} / 100 \mathrm{~mA}$ linear regulator and a $1.2 \mathrm{~V} / 10 \mathrm{~A}$ switching regulator. In addition, the start-up of all the supplies must rise monotonically during $\mathrm{t}_{\text {RAMP }}\left(50 \mu \mathrm{\mu S} \leq \mathrm{t}_{\text {RAMP }} \leq 50 \mathrm{~ms}\right.$ ). Thus, a Soff-Start
$(\mathrm{SS})$ is ferrite bead for avoiding switching noise to enter the PLLs.

PCB Details

The PCB has been manufactured in a class 6 standard using a 10-layer stack-up that contans two power planes, two ground planes and six signal layers. Trace impedance. The power planes have been geographically split in islands
for allocating in voltage references for allocating in vo
where needed.

\section{Class 6 PCB}

$125 \mu \mathrm{m}$ etch width and $125 \mu \mathrm{m}$ etch spacing

$200 \mu \mathrm{m}$ via diameter

$250 \mu \mathrm{m}$ via-etch spacing
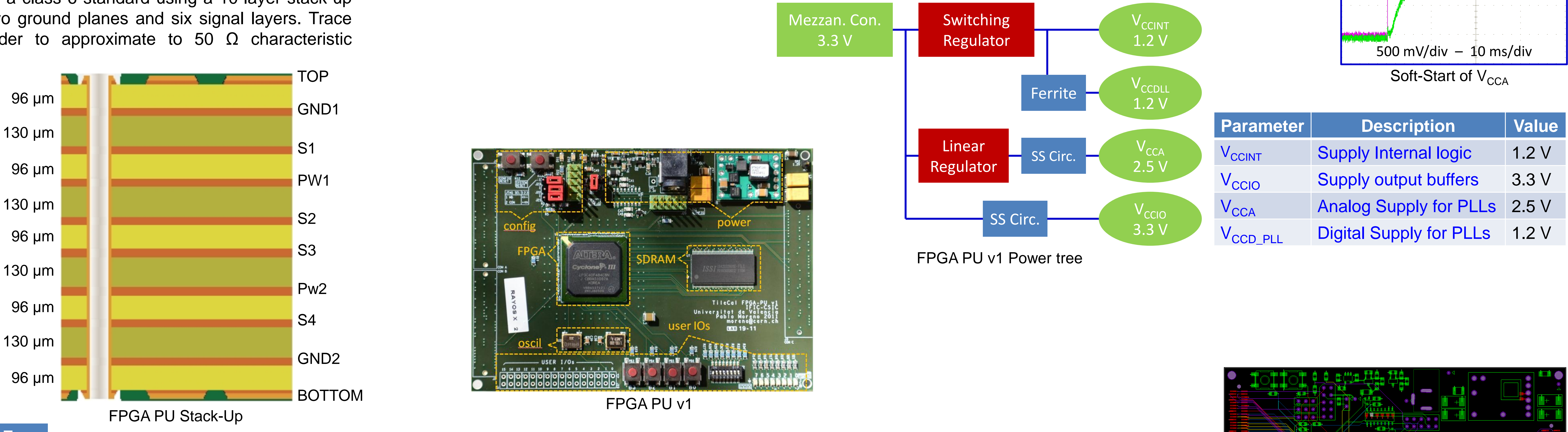

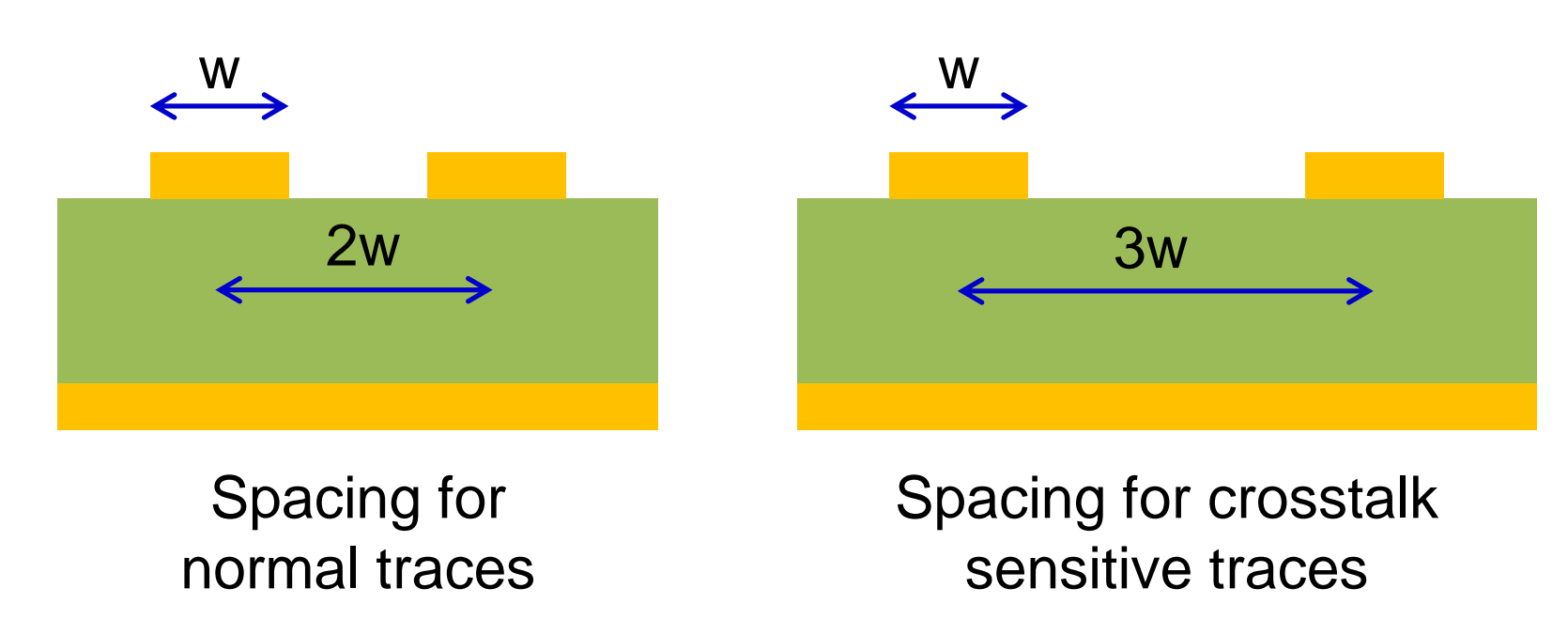

PCB Routing

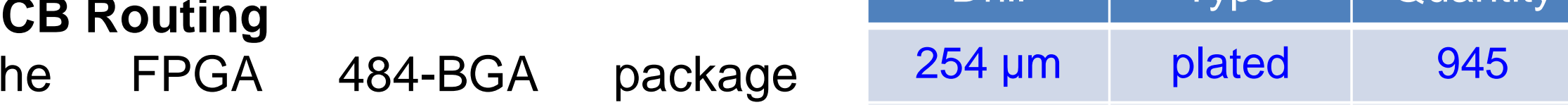
introduces certain complexity in the PCB routing process. A total of 280 using 630 etch traces and 1030 vias.

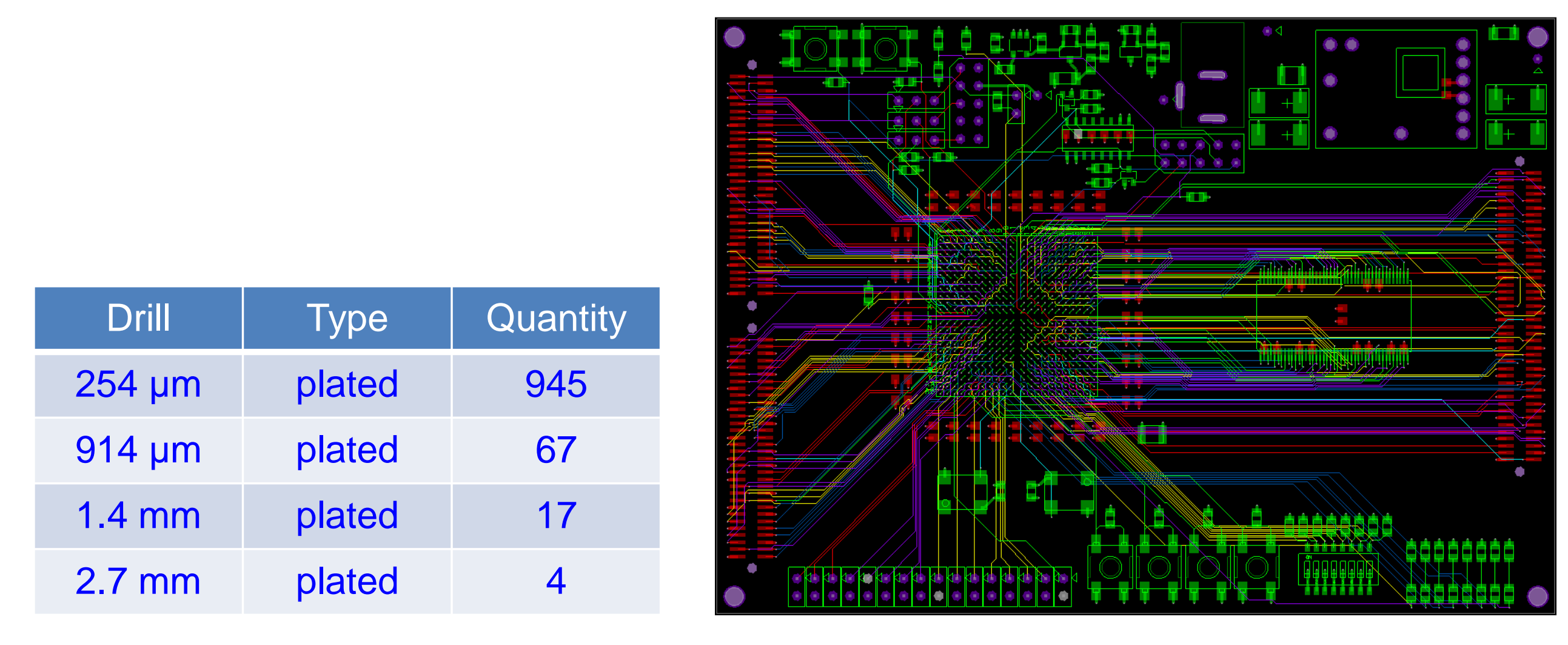

Firmware Development

Phase 1

The first versions of the firmware have been developed in order to check the proper operation of all the basic
elements on the board in standalone mode. The FPGA tarts up and is configured correctly. The configuration buttons and all the user interfaces work as expected.

Phase 2

In this phase, the firmware is being developed for the

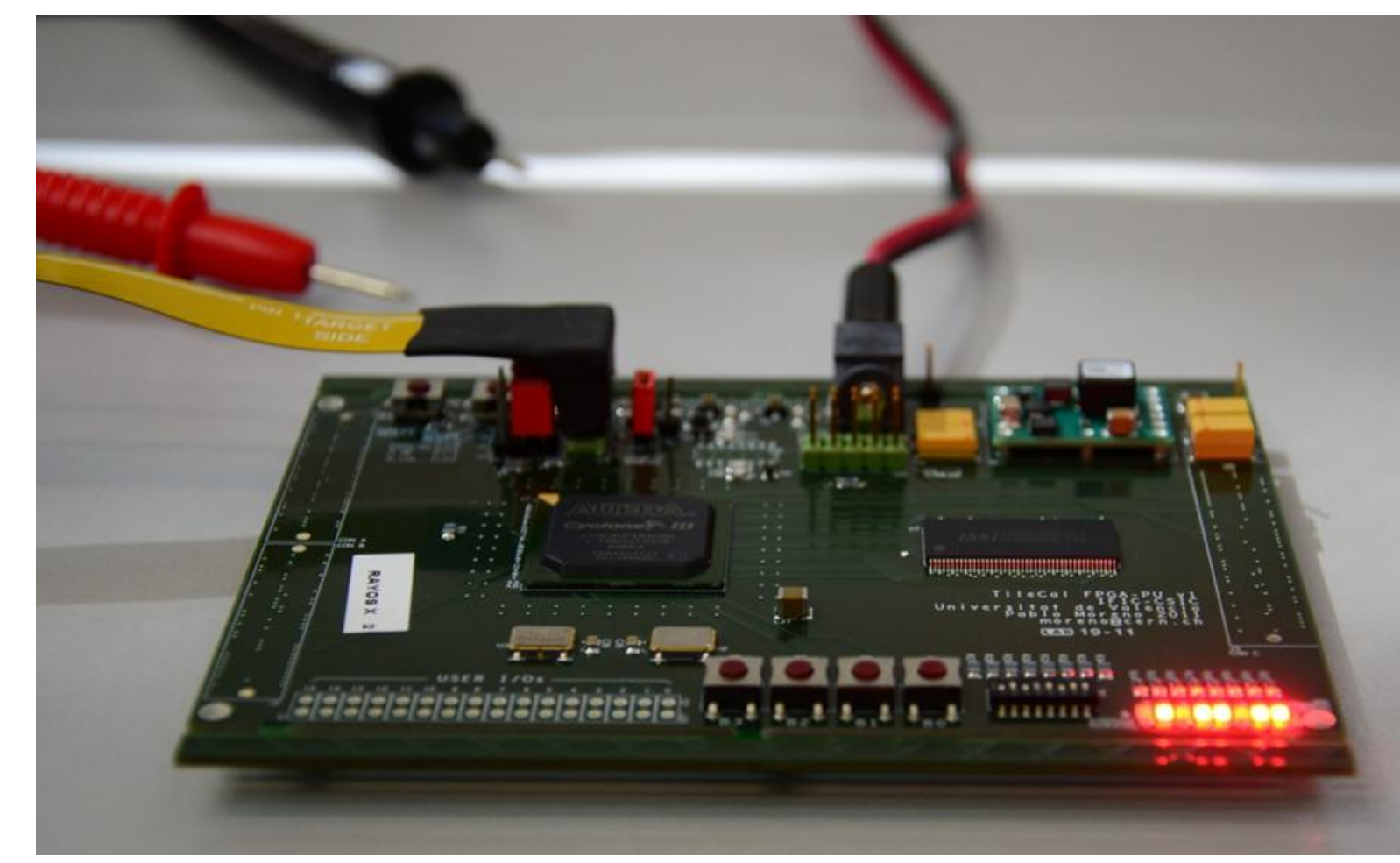

The target is to be fully compatible with the present DSP slices and interfacing this implementation with the
system. The functionalities of the different parts of the rest of functional blocks are the main goals in this phase. DSP PU have to be implemented on the cyclone III In Phase 3, the extended functionality of the system will of the firmware implemented in this phase, permit programmable logic and DSP support. A Nios II-based backplane of the ROD crate the FPGA PU V1 from the SOPC Future Work The most immediate work is to complete the Phase 2.
The implementation of the OF algorithm on the FPGA advantage of the possibility to use $\mathrm{C}++$ programs instead
of $H D L$ code, thus simplifying the performance of such of $\mathrm{HDL}$ code, thus simplifying the performance of such 